\title{
PRODUCT FORMULAS, HYPERGROUPS, AND THE JACOBI POLYNOMIALS
}

\author{
WILLIAM C. CONNETT AND ALAN L. SCHWARTZ
}

If $\mathscr{P}=\left\{p_{n}\right\}_{n \in \mathbf{N}_{0}}\left(\mathbf{N}_{0}=\{0,1,2, \ldots\}\right)$ is a sequence of orthogonal functions on a real interval $I$, we say that $\mathscr{P}$ has a product formula if for each $s, t$ in $I$, there is a Borel measure $\mu_{s, t}$ with $\operatorname{supp}\left(\mu_{s, t}\right) \subseteq I$ such that

$$
\int_{I} p_{n} d \mu_{s, t}=p_{n}(s) p_{n}(t)
$$

for every $n$ in $\mathbf{N}_{0}$. Such formulas are important because they give rise to a variety of measure algebras and the means to study their harmonic analysis. An important class of such formulas was established by Gasper [8] for the Jacobi polynomials $P_{n}^{(\alpha, \beta)}(x)$ which are orthogonal on $[-1,1]$ with respect to the weight $(1-x)^{\alpha}$ $\times(1+x)^{\beta} d x$. These include Chebyshev, Legendre, and ultraspherical or Gegenbauer polynomials as special cases. The product formula for Jacobi polynomials has 1 as an identity in the sense that for all $t$ in $[-1,1] \mu_{1, t}$ is the unit point mass concentrated at $t$, and it has continuous support in the sense that $\operatorname{supp}\left(\mu_{s, t}\right)$ is a continuous function of $(s, t)$. Moreover, the measures $\mu_{s, t}$ are all positive if and only if

$$
\alpha \geq \beta>-1 \text { and either } \beta \geq-1 / 2 \text { or } \alpha+\beta \geq 0 .
$$

It is natural to ask which orthogonal polynomials have such product formulas. The answer is a converse to Gasper's result:

Theorem 1. If a family $\mathscr{P}$ of orthogonal polynomials has a product formula with identity, continuous support, and nonnegative measures $\mu_{s, t}$ then up to a linear change of variables, the members of $\mathscr{P}$ are Jacobi polynomials with parameters $\alpha$ and $\beta$ satisfying equation (2).

Received by the editors January 25, 1989 and, in revised form, July 11, 1989.

1980 Mathematics Subject Classification (1985 Revision). Primary 33A65; Secondary $43 \mathrm{~A} 10,42 \mathrm{C} 05$.

Key words and phrases. Jacobi Polynomials, hypergroups, measure algebras. 
The proof of Theorem 1, as well as some of its applications will require the notion of hypergroups. A hypergroup is a measure algebra more general then the convolution measure algebra associated with a group (for instance, a convolution of point masses need not be a point mass), but with enough structure to make harmonic analysis possible.

To be precise, let $H$ be a locally compact Hausdorff space and let $M(H)$ denote the bounded Borel measures on $H$; if $\mu \in M(H), \operatorname{supp}(\mu)$ is the support of $\mu$. The unit point mass concentrated at $s$ is indicated by $\delta_{s} ; C(H)$ is the space of continuous complex-valued functions on $H$; and $C_{c}(H)$ consists of all $f$ in $C(H)$ with compact support.

If $M(H)$ is a Banach algebra with multiplication * (called a convolution), then $(H, *)$ is a hypergroup if the following axioms are satisfied:

(1) A convolution of probability measures is a probability measure.

(2) The mapping $(\mu, \nu) \rightarrow \mu * \nu$ is continuous from $M(H) \times$ $M(H)$ into $M(H)$ where $M(H)$ is given the weak topology with respect to $C_{c}(H)$.

(3) There is an element $e \in H$ such that $\delta_{e} * \mu=\mu * \delta_{e}=\mu$ for every $\mu \in M(H)$.

(4) There is a homeomorphic mapping $s \rightarrow s^{\vee}$ of $H$ into itself such that $s^{\vee \vee}=s$ and $e \in \operatorname{supp}\left(\delta_{s} * \delta_{t}\right)$ if and only if $t=s^{\vee}$. by

(5) For $\mu, \nu \in M(H)(\mu * \nu)^{\vee}=\nu^{\vee} * \mu^{\vee}$ where $\mu^{\vee}$ is defined

$$
\int_{H} f(s) d \mu^{\vee}(s)=\int_{H} f\left(s^{\vee}\right) d \mu(s) .
$$

(6) The mapping $(s, t) \rightarrow \operatorname{supp}\left(\delta_{s} * \delta_{t}\right)$ is continuous from $H \times$ $H$ into the space of compact subsets of $H$ as topologized in [14]. See [5, 6, 9 and 16] for more about hypergroups.

There is a natural connection between product formulas and hypergroups. Suppose the hypotheses of Theorem 1 are satisfied. Define a product $*$ on $M(I)$ by

$$
\int_{I} f d(\nu * \lambda)=\int_{I} \int_{I}\left(\int_{I} f d \mu_{s, t}\right) d \nu(s) d \lambda(t),
$$

then $(I, *)$ is a hypergroup if we require the additional conditions: (i) there is $e \in I$ such that $p_{n}(e)=1 \quad\left(n \in \mathbf{N}_{0}\right)$, and (ii) $0 \in$ $\operatorname{supp}\left(\mu_{s, t}\right)$ if and only if $s=t$ [16]. $\mathscr{P}$ is the set of characters for this hypergroup in the sense that (1) and (3) imply

$$
\int_{I} p_{n} d(\nu * \lambda)=\left(\int_{I} p_{n} d \nu\right)\left(\int_{I} p_{n} d \lambda\right)\left(n \in \mathbf{N}_{0}\right) .
$$


The hypergroups arising from the ultraspherical polynomials are useful in studying certain stochastic processes on the sphere (see also [9]).

Theorem 1 immediately yields the following characterizations of two classes of hypergroups (see [5] for definitions):

Theorem 2. If $(H, *)$ is a hypergroup with $H$ a real interval which has polynomial characters of every degree, then up to a linear change of variables, $(H, *)$ is one of the Jacobi polynomial hypergroups $(H, * ; \alpha, \beta)$ with parameters $\alpha$ and $\beta$ satisfying equation (2).

Theorem 3. The only strong polynomial hypergroups (see [9]) are those that arise from the Jacobi polynomials with parameters $\alpha$ and $\beta$ satisfying (2).

Theorem 3 gives further credence to Heyer's remark $[9,3.7]$ that strong hypergroups are fairly rare. Thus results for strong polynomial hypergroups [11, Theorem 4], or for hypergroups with polynomial characters are no more than the corresponding results for the hypergroups arising from Jacobi polynomials for which there is already an extensive literature; e.g., [3, 4], and the references cited there. It is possible to give explicit formulas for the parameters $\alpha$ and $\beta$ and the $p_{n}$. We first observe that one consequence of Theorem 1 is that $e$ must be one of the endpoints of $I$ [16]:

Theorem 4. Let $\mathscr{P}$ satisfy the hypotheses of Theorem 1 with identity $e$; let $a$ be the other endpoint of $I$, and let $d_{n}=\frac{1}{2} p_{n}^{\prime}(e) /$ $(e-a)$, then $\alpha=\left(d_{2}-2 d_{1}\right)^{-1}-1, \beta=\left(2 d_{1}-1\right)\left(d_{2}-2 d_{1}\right)^{-1}-1$, and $p_{n}(t)=R_{n}^{(\alpha, \beta)}(t)=\left(\begin{array}{c}n+\alpha \\ n\end{array}\right)^{-1} p_{n}^{(\alpha, \beta)}\left(\frac{a+e-2 t}{a-e}\right)$.

We note that there are systems of orthogonal polynomials besides the Jacobi polynomials which have product formulas.

1. The generalized Chebyshev polynomials have a product formula [10] which does not have support continuity since $\operatorname{supp}\left(\mu_{1,-1}\right)=\{-1\}$ but $\operatorname{supp}\left(\mu_{t,-t}\right)=\left[-1,1-2 t^{2}\right] \cup\left[2 t^{2}-1,1\right]$ (see [12, p. 207]; (in that article, the notation for $\mu_{s, t}$ is $p_{s} * p_{t}$ ).

2. The continuous $q$-Jacobi polynomials $[15]$ on $H=[-1,1]$ have a product formula with a nonnegative absolutely continuous measure for all $s, t \in H$ and the support of the measure is always all of $H$, hence there can be no identity.

Outline of proof of Theorem 1. The proof has two parts. First a technique inspired by [13] and exploited in [5] shows that the members of $\mathscr{P}$ are the eigenfunctions of a second order linear differential operator. That is $y=p_{n}(t)$ satisfies

$$
q y^{\prime \prime}+p y^{\prime}=\lambda_{n} y
$$


with $\lambda_{n}=p_{n}^{\prime}(e), p(t)=p_{1}(t), q(t)=\left[\lambda_{2} / p_{2}^{\prime \prime}\right]\left[p_{2}(t)-p_{1}(t)\right]-$ $\left[p_{1}(t) / \lambda_{1}\right]\left[p_{1}(t)-1\right]$. Secondly we employ a result of Bochner [2] to show that the differential equation must in fact be the one associated with the Jacobi polynomials.

If $q \equiv 0$, the solutions of equation (4) are $p_{n}(x)=x^{n}$ which are not orthogonal on any interval since these polynomials do not have simple zeros (see [18, Theorem 3.3.1]).

If $q$ is a nonzero constant, $p_{n}(x)=H_{n}(x) / H_{n}(e)$, where $H_{n}$ is the $n$th degree Hermite polynomial. The condition $q^{\prime \prime}=0$ entails

$$
p_{2}^{\prime}(e)=2 p_{1}^{\prime}(e)
$$

which leads to a contradiction when one attempts to solve equation (5) for $e$.

If $q$ has degree exactly one then $p_{n}$ is the normalized Laguerre polynomial $L_{n}^{\alpha}(x) / L_{n}^{\alpha}(e)$. Once more, equation (5) must hold, but this time it can be solved to obtain $e=0$. The nonexistence of a product formula in this case follows from [1], (Theorem 6 and the remarks following).

Thus $q$ must have degree exactly two. A linear change of variables transforms equation (4) into one of the forms

$$
\begin{gathered}
x^{2} y^{\prime \prime}+(\delta+\varepsilon x) y^{\prime}+\lambda y=0 . \\
x(1-x) y^{\prime \prime}+(\delta+\varepsilon x) y^{\prime}+\lambda y=0 .
\end{gathered}
$$

We shall eliminate the possibility of equation (6) by showing that if the differential operator $L=t^{2}\left(d^{2} / d t^{2}\right)+(\delta+\varepsilon t) d / d t$ has polynomial eigenvectors, they cannot be orthogonal. Bochner [2] considers two cases: $\delta=0$ and $\delta \neq 0$.

If $\delta=0$, then $L$ has polynomial eigenfunctions provided $\varepsilon=$ $1-k, k=1,2, \ldots$, in which case the eigenfunctions are of the form $p_{n}(x)=a_{n} x^{n}+b_{n} x^{k-n}$. These cannot be orthogonal polynomials since for $n>k$, the zeros of $p_{n}$ are not distinct (cf. [18, Theorem 3.3.1]).

If $\delta \neq 0$, it is no loss of generality to consider only $\delta+\varepsilon t=$ $(k+1) t-1$. Then $L$ has polynomial eigenfunctions unless $k$ is a negative integer, and if $-k \notin \mathbf{N}_{0}$ is fixed, these are given by

$$
P_{n}(t)=\sum_{\nu=0}^{n} \nu !\left(\begin{array}{l}
n \\
\nu
\end{array}\right)\left(\begin{array}{c}
-n-k \\
\nu
\end{array}\right) t^{\nu} .
$$

It can be shown that these polynomials are not orthogonal because they do not satisfy an appropriate three-term recurrence (cf. [17, Theorem 1]). 
Having eliminated all other possibilities, we conclude that the differential equation (4) must have been transformed into equation (7). If in that equation we make the change of variables $x=2 t-1$, we obtain a differential equation satisfied by $y=P_{n}^{(\alpha, \beta)}(x)$ (see [18, equation (2.1)]) with $\alpha=-2 \varepsilon-2 \delta-1, \beta=2 \delta-1$, and $\lambda=n(n+\alpha+\beta+1) / 4$, and the proof is complete.

Remark. Slight modifications can be made in the proof to allow the hypotheses of Theorem 1 to be weakened as follows:

1. Instead of assuming that $e$ is an identity, it is enough to ask that for each $t \in I, \mu_{e, t}$ be concentrated on a single point. (It is not necessary to require that $\mu_{e, t}$ be a unit mass or concentrated on the point $t$.)

2. The support continuity may be replaced by

$$
\lim _{s \rightarrow e}\left(\operatorname{diam}\left(\operatorname{supp} \mu_{s, t}\right)\right)=0 .
$$

3. The combination of support continuity and nonnegativity may be replaced by the single condition $\int_{I}(r-t)^{n} d \mu_{s, t}(r)=o(s-e)$ for $n>2$.

4. The condition that the polynomials satisfy a product formula can be replaced by the assumption that the weighted polynomials $m(t) p_{n}(t)$ satisfy a product formula, where $m(t)$ is a fixed positive function on $I$.

Proof of Theorems 2 and 3. The hypotheses of these theorems are by definition stronger than the hypotheses of Theorem 1, since the characters of a commutative hypergroup are necessarily orthogonal [6, Theorem 3.5]. The range for the parameters is the intersection of those given by Gasper in his studies [7, and 8] of the two Jacobi convolution structures.

Proof of Theorem 4. The linear transformation $x=\phi(t)=$ $(a+e-2 t) /(a-e)$ maps $I$ onto $[-1,1]$ and carries $e$ to 1 . Thus $p_{n}(t)=R_{n}^{(\alpha, \beta)}(\phi(t))$, and the relations are obtained by referring to the explicit formulas for the first two moments associated with Jacobi polynomials as given in [5, equations (1.10) and (1.11)].

\section{REFERENCES}

1. R. Askey, Orthogonal polynomials and positivity, Studies in Applied Mathematics 8, Wave Propagation and Special Functions, SIAM, 64-85 (1970).

2. S. Bochner, Über Sturm-Liouvillische Polynomesysteme, Math. Z. 29 (1929), 730-736.

3. W. C. Connett and A. L. Schwartz, A multiplier theorem for Jacobi expansions, Studia Math. 52 (1975), 243-261. 
4. __ The Littlewood-Paley theory for Jacobi expansions, Trans. Amer. Math. Soc. 251 (1979), 219-234.

5. __ Analysis of a class of probability preserving measure algebras on compact intervals, Trans. Amer. Math. Soc. (to appear).

6. C. F. Dunkl, The measure algebra of a locally compact hypergroup, Trans. Amer. Math. Soc. 179 (1973), 331-348.

7. G. Gasper, Linearization of the product of Jacobi polynomials. II, Canad. J. Math. 32 (1970), 582-593.

8. __ Banach algebras for Jacobi series and positivity of a kernel, Ann. of Math. 95 (1972), 261-280.

9. H. Heyer, Probability theory on hypergroups: a survey, Probability Measures on Groups VII, Proceedings Oberwolfach 1983, Lecture Notes in Math., vol. 1064, Springer-Verlag, Berlin and New York, 1984.

10. T. P. Laine, The product formula and convolution structure for the generalized Chebyshev polynomials, SIAM J. Math. Anal. 11 (1980), 133-146.

11. R. Lasser, Bochner theorems for hypergroups and their applications to orthogonal polynomial expansions, J. Approximation Theory 37 (1983), 311-325.

12. $\frac{1}{209}$, Orthogonal polynomials and hypergroups, Rend. Mat. (7) 3 (1983), 185209.

13. B. M. Levitan, Generalized translation operators, Israel Program for Scientific Translations, Jerusalem, 1964.

14. E. Michael, Topologies on spaces of subsets, Trans. Amer. Math. Soc. 71 (1951), 152-182.

15. M. Rahman, A product formula for the continuous q-Jacobi polynomials, J. Math. Anal. Appl. 118 (1986), 309-322.

16. A. L. Schwartz, Classification of one-dimensional hypergroups, Proc. Amer. Math. Soc. 103 (1988), 1073-1081.

17. J. Shohat, The relation of the classical orthogonal polynomials to the polynomials of Appell, Amer. J. Math. 58 (1936), 453-464.

18. G. Szego, Orthogonal polynomials, Amer. Math. Soc. Colloq. Publ. vol. 23, Amer. Math. Soc., Providence, R.I., 1939.

Department of Mathematics and Computer Science, University of Missouri, St. Louis, Missouri 63121-4499 